\title{
Dahlia merckii y D. rupicola (Asteraceae, Coreopsideae), dos nuevos registros para la región de la Nueva Galicia, México
}

\section{Dahlia merckii and D. rupicola (Asteraceae, Coreopsideae), two new records for the Nueva Galicia region, Mexico}

\author{
Rafael Guadalupe Macías-Flores (D), Claudia Janeth Ramírez-Díaz² (D, Jesús Guadalupe González-Gallegos³ (D) \\ Pablo Carrillo-Reyes ${ }^{4}$ (D), Arturo Castro-Castro ${ }^{3,5}$ (D)
}

1 Tecnológico Nacional de México, Instituto Tecnológico de Altamira, Licenciatura en Biología, carretera Tampico-Mante km 24.5, 89600 Altamira, Tamaulipas, México.

2 Centro de Investigación Científica de Yucatán, A.C. (CICY), calle 43 No. 130, Col. Chuburná de Hidalgo, 97205 Mérida, Yucatán, México.

3 Cátedras CONACYT-Instituto Politécnico Nacional, Centro Interdisciplinario de Investigación para el Desarrollo Integral Regional, Sigma No. 119, 20 de Noviembre II, 34234 Durango, Durango, México.

4 Universidad de Guadalajara, Centro Universitario de Ciencias Biológicas y Agropecuarias, Herbario Luz María Villarreal de Puga del Instituto de Botánica, Camino Ramón Padilla Sánchez No. 2100, Nextipac, 44600 Zapopan, Jalisco, México.

5 Autor para la correspondencia: arca68@hotmail.com

Recibido: 11 de diciembre de 2017 Revisado: 9 de febrero de 2018. Aceptado: 2 de abril de 2018

Primero en línea: 16 de agosto de 2018 Publicado: 3 de octubre de 2018

\section{Citar como:}

Macías-Flores, R. G., C. J. Ramírez-Díaz, J. G. González-Gallegos,P.Carrillo-Reyesy A. Castro-Castro. 2018. Dahlia merckii y D. rupicola (Asteraceae, Coreopsideae), dos nuevos registros para la región de la Nueva Galicia, México. Acta Botanica Mexicana 125: 49-60. DOl: 10.21829/ abm125.2018.1328

DOI:

10.21829/abml25.2018.1328

\section{Resumen:}

Antecedentes y Objetivos: Dahlia es un género característico de Asteraceae tribu Coreopsideae y para la Nueva Galicia se conocían ocho especies. El presente texto tiene como finalidad reportar dos especies adicionales.

Métodos: Exploraciones botánicas en Nueva Galicia fueron llevadas a cabo. Se revisaron y determinaron ejemplares depositados en los herbarios ANSM, BUAP, CFNL, CHAPA, CIIDIR, ENCB, FCME, IBUG, IEB, MEXU, QMEX, SLPM, UAT, UAZ y XAL. Se realizaron descripciones morfológicas ampliadas y una clave de identificación a partir de la consulta de literatura especializada, del análisis de ejemplares de herbario y la revisión de plantas en el campo. La información recopilada se analizó con un sistema de información geográfica para la elaboración de mapas de distribución y propuestas para categorías de protección.

Resultados clave: Se documenta a Dahlia merckii y D. rupicola para la Nueva Galicia. Estos nuevos registros extienden el área de distribución para ambas especies al occidente de México. De acuerdo a las categorías y criterios de la Unión Internacional para la Conservación de la Naturaleza, D. merckii se puede tratar como una especie en Preocupación Menor. Dahlia rupicola podría considerarse En Peligro tomando en cuenta los valores de extensión de la ocurrencia y el área de ocupación.

Conclusiones: Estos datos extienden la amplitud adaptativa de las dos especies de Dahlia a hábitats con condiciones ecológicas diferentes a las antes reconocidas. Recomendamos la realización de estudios para evaluar el estado de conservación de la totalidad de especies del género, de acuerdo a las categorías de conservación a nivel nacional e internacional. Por último, la ampliación de las distribuciones de $D$. merckii y $D$. rupicola también señala la necesidad de continuar e impulsar la exploración botánica en México.

Palabras clave: disyunción geográfica, Heliantheae, occidente de México.

\section{ABSTRACT:}

Background and Aims: Dahlia is a characteristic genus of Asteraceae tribe Coreopsideae and eight species were known in Nueva Galicia. The objective of the present communication is to report two additional species.

Methods: Botanical explorations were carried out in Nueva Galicia. Additionally, Dahlia specimens deposited at the herbaria ANSM, BUAP, CFNL, CHAPA, CIIDIR, ENCB, FCME, IBUG, IEB, MEXU, QMEX, SLPM, UAT, UAZ and XAL were reviewed and identified. Morphological descriptions and an identification key were made based on the analysis of specialized literature, revision of herbaria specimens and plants in the field. A database was compiled with the information and was analyzed with a geographic information system for the elaboration of distribution maps and proposals for protection categories.

Key results: We documented Dahlia merckii and D. rupicola in Nueva Galicia. These new records extend the distribution area for both species to western Mexico. According to the categories and criteria of the International Union for Conservation of Nature, D. merckii can be qualified as a Least Concern species. Meanwhile, D. rupicola could be considered as Endangered if the area of occupation and the extension of occurrence are taken into account.

Conclusions: This information extends the adaptive amplitude of both Dahlia species to habitats with different ecological conditions to those previously documented. We recommend carrying out studies to evaluate the conservation status of all the species in the genus according to conservation categories at national and international scales. Finally, the expanded distributions of D. merckii and D. rupicola indicate the need to continue and promote botanical exploration in Mexico.

Key words: geographic disjunction, Heliantheae, western Mexico. 


\section{INTRODUCCIÓN}

En la región de la Nueva Galicia confluyen seis provincias biogeográficas: Costa Pacífica Mexicana, Cuenca del Balsas, Desierto Chihuahuense, Faja Volcánica Transmexicana, Sierra Madre del Sur y Sierra Madre Occidental (Rzedowski y McVaugh, 1966; Morrone et al., 2017). Esta coincidencia de sistemas biogeográficos ha promovido una riqueza florística elevada. La familia Asteraceae es un ejemplo de esta diversificación en el occidente de México. Por ejemplo, Villaseñor (1991; 1993) y Harker (2017) señalan que en el occidente mexicano existen entre 690 y 889 taxones para la familia, y que esta región del país representa uno de sus centros de riqueza y endemismo para las tribus Heliantheae y Coreopsideae.

Uno de los integrantes de Coreopsideae es el género Dahlia Cav. El género se integra por 37 especies y para la Nueva Galicia se han reportado ocho de ellas: $D$. barkerae Knowles \& Westc., D. coccinea Cav., D. pugana Aarón Rodr. \& Art. Castro, D. scapigera (A. Dietr.) Knowles \& Westc., D. sherffii P.D. Sørensen, D. sorensenii H.V. Hansen \& Hjert., D. tenuicaulis P.D. Sørensen y D. wixarika Art. Castro, Carr.-Ortiz \& Aarón Rodr. (Sørensen, 1969; McVaugh, 1984; Castro-Castro et al., 2012, 2015; Carrasco-Ortiz, 2013). Sin embargo, exploraciones botánicas y la revisión de ejemplares de herbario recientes nos han permitido identificar una riqueza mayor en la Nueva Galicia.

El objetivo de esta comunicación es dar a conocer la presencia de D. merckii Lehm. y D. rupicola P.D. Sørensen. Se presentan descripciones morfológicas ampliadas para estas especies, un mapa y discusiones sobre su distribución geográfica, su estado de conservación, y una clave para la identificación de todas las especies de Dahlia para la región de estudio.

\section{Materiales y MÉtodos}

Se realizaron exploraciones botánicas en la región de la Nueva Galicia. Se examinaron y determinaron ejemplares depositados en los herbarios ANSM, BUAP, CFNL, CHAPA, CIIDIR, ENCB, FCME, IBUG, IEB, MEXU, QMEX, SLPM, UAT, UAZ y XAL (acrónimos según
Thiers, 2017). Las descripciones morfológicas ampliadas y la clave de identificación se lograron a partir de la consulta de literatura especializada (Sørensen, 1969; Rzedowski y Calderón de Rzedowski, 2008), revisión de ejemplares tipo (JSTOR, 2017), del análisis de 148 ejemplares de herbario y observación de plantas en campo. Para los términos botánicos se utilizó la propuesta de Moreno (1984). Se conformó una base de datos que se analizó con el sistema de información geográfica ArcView Gis 3.3 (ESRI, 2002) para la elaboración de mapas de distribución de las especies dentro de los límites de la Nueva Galicia bajo las pautas señaladas en Castro-Castro et al. (2015). Por último, para el área de distribución de Dahlia merckii y D. rupicola se calculó la extensión de la presencia (EOO) y el área de ocupación (AOO) con el uso de la plataforma GeoCAT (Bachman et al., 2011). Los valores resultantes, junto con nuestras observaciones de campo, fueron utilizados para determinar su riesgo de extinción con base en el sistema de clasificación de la Unión Internacional para la Conservación de la Naturaleza (UICN, 2012).

\section{Resultados}

Dahlia tiene una distribución amplia en la Nueva Galicia (Fig. 1). Además de las ocho especies citadas por CastroCastro et al. (2015), D. merckii y D. rupicola representan dos nuevos registros y elevan la cantidad de taxones a diez. A continuación, se presenta una clave para la identificación para las especies de Dahlia en la región de estudio, descripciones morfológicas ampliadas y comentarios sobre la distribución y estado de conservación para los dos nuevos registros.

\section{Clave para la identificación de las especies de} Dahlia que crecen en Nueva Galicia, México

1a. Filarias externas reflejas o extendidas en la antesis ...2

1b. Filarias externas erectas o ascendentes en la antesis...6 6

2a. Flores liguladas, color rojo, anaranjado o amarillo ... D. coccinea Cav.

2b. Flores liguladas, color violeta ................................. 3

3a. Plantas arborescentes o arbustivas, decumbentes, 1.5-3 $\mathrm{m}$ de altura ......... D. tenuicaulis P.D. Sørensen 

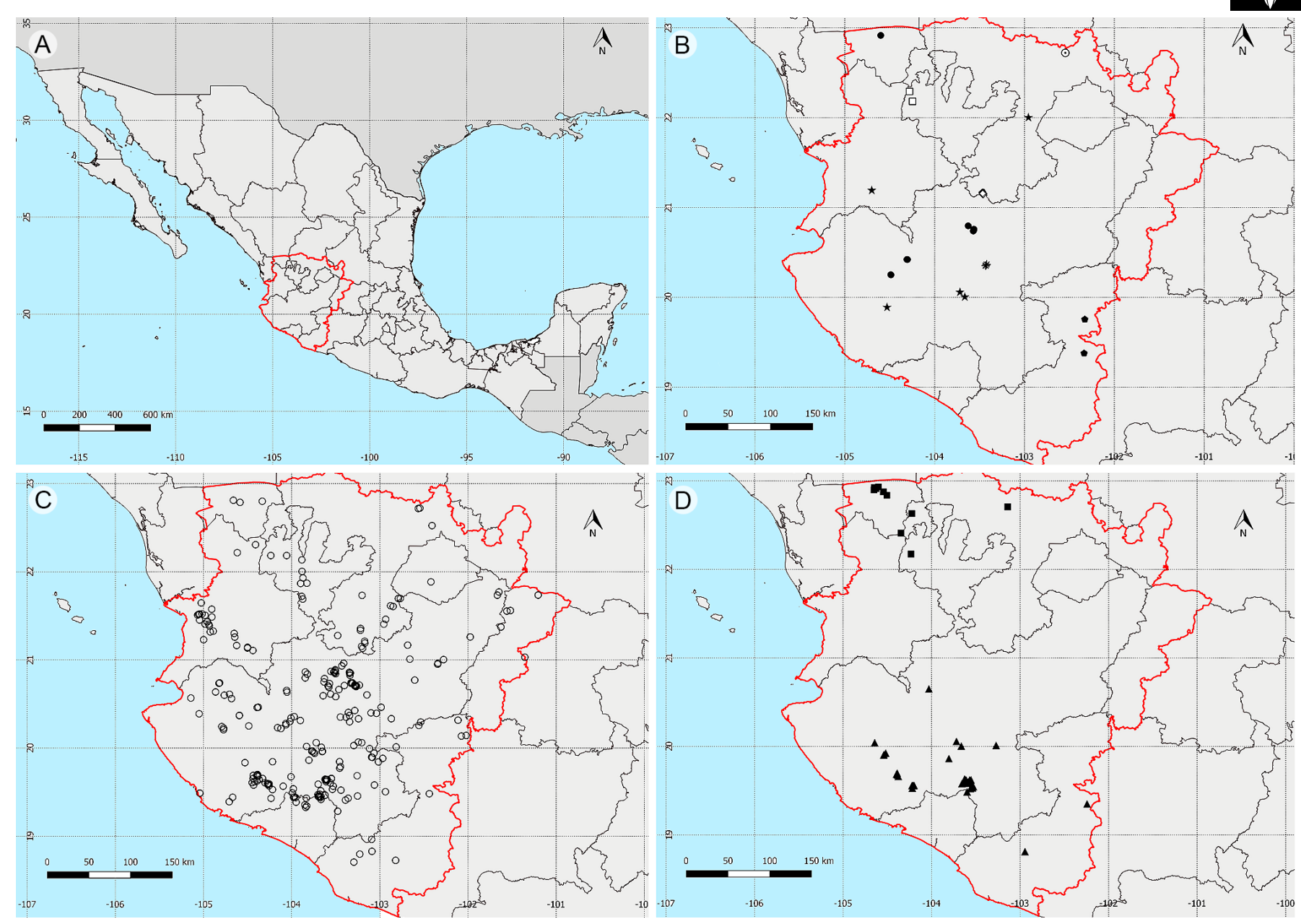

Figura 1: Distribución geográfica del género Dahlia Cav. en la Nueva Galicia, México. A. mapa de México con el polígono de la Nueva Galicia; B. D. barkerae Knowles \& Westc. (asteriscos), D. merckii Lehm. (círculos vacíos con punto central), D. pugana Aarón Rodr. \& Art. Castro (círculos rellenos), D. rupicola P.D. Sørensen (rombos vacios), D. scapigera (A. Dietr.) Knowles \& Westc. (pentágonos), D. sorensenii H.V. Hansen \& Hjert. (estrellas), D. wixarika Art. Castro, Carr.-Ortiz \& Aarón Rodr. (cuadros vacíos); C. D. coccinea Cav. (círculos vacios); D. D. sherffi P.D. Sørensen (cuadros rellenos), D. tenuicaulis P.D. Sørensen (triángulos).

3b. Plantas herbáceas, erectas, $0.4-2 \mathrm{~m}$ de altura 4

4a. Pecíolos huecos; foliólulos alternos

D. merckii Lehm.

4b. Pecíolos sólidos; foliólulos opuestos 5

5a. Hojas membranáceas; pecíolo, raquis y peciólulos no alados; flores del disco 50-70

D. sherffii P.D. Sørensen

5b. Hojas rígidas y coriáceas; pecíolo, raquis y peciólulos alados; flores del disco 90-145

D. sorensenii H.V. Hansen \& Hjert.

6a. Pecíolo y raquis alados y aplanados 7

6b. Pecíolo y raquis no alados y conduplicados 8 7a. Tallos y envés de las hojas vilosos; folíolos anchamente ovados a suborbiculares; capítulos dispuestos en grupos de 2-3 ...... D. barkerae Knowles \& Westc.

7b. Tallos y hojas glabras o glabriúsculos; folíolos romboides a lanceolados; capítulos solitarios

D. scapigera (A. Dietr.) Knowles \& Westc.

8a. Plantas arbustivas; raíces tuberosas ausentes; hojas bipinnatisectas; cipselas oblongo-rectangulares, 10$12 \mathrm{~mm}$ de longitud D. rupicola P.D. Sørensen

8b. Plantas herbáceas; raíces tuberosas presentes; hojas pinnadas o bipinnadas; cipselas elípticos, 6-9 $\mathrm{mm}$ de longitud, obovados u oblongo-lanceolados 9 
9a. Hojas pinnadas, bipinnadas o enteras; foliólulos opuestos; estigmas de las flores liguladas 2-furcados; cipselas lisas; vilano discoide o ausente

D. pugana Aarón Rodr. \& Art. Castro

9b. Hojas pinnadas o bipinnadas; foliólulos alternos; estigmas de las flores liguladas 2-3-furcados; cipselas tuberculadas; vilano ausente

.. D. wixarika Art. Castro, Carr.-Ortiz \& Aarón Rodr.

Dahlia merckii Lehm., Del. Sem. Hort. Hamburg. 4. 1839. TIPO: MÉXICO. Localidad exacta desconocida, material original cultivado en el Jardín Botánico de Hamburgo a partir de semillas colectadas en México, H. J. Merck s.n. (lectotipo: GH!, isolectotipo: K!). Figs. 1B, 2C.

Hierba perenne, 0.5-2 $\mathrm{m}$ de alto; raíces fasciculadas; tallo herbáceo, fistuloso, 0.3-1.2 cm de diámetro, rollizo o hexangular en corte transversal, glabro o con anillos de tricomas en los nudos; internudos 1-27 cm de largo; pecíolo hueco, 4-16 ×0.2 cm, ensanchado en la base y envolviendo al tallo, glabro; hojas deltoides a ovadas en contorno general, $14-38 \times 12-35 \mathrm{~cm}$, pinnadas o frecuentemente bipinnadas, a veces algunas indivisas; estipelas presentes en la base del primer par de folíolos, 0.8$4.6 \times 0.3-3.2 \mathrm{~cm}$; folíolos 5-7, opuestos, sésiles, (2.5-)4$16 \times 1.7-5.3 \mathrm{~cm}$, a veces el folíolo terminal con peciólulo, (1-)2.8-4 cm de largo, ovados, obovados o romboides, agudos o acuminados en el ápice, cuneados y decurrentes en la base, herbáceos, bicolores, color verde oscuro en el haz, verde pálidos en el envés, glabros o esparcidamente pubérulos en ambas superficies, margen irregularmente serrado a dentado-crenado, con 1-5 dientes por lado, a veces lobado o subentero, ciliolado; lóbulos de los folíolos y foliólulos alternos, 0.6-2.7 × 0.9-1.3 cm, lanceolados; cabezuelas $10-90$ por planta, $4.5-6 \mathrm{~cm}$ de diámetro, elevadas por encima de la parte foliosa del tallo en numerosas ramas; pedúnculos (0.3-)1-30 cm de largo, glabros; involucro anchamente campanulado, filarias externas $5(-7)$, extendidas o reflejas en la antesis, color verde, lineares a subuladas, lanceoladas o espatuladas, glabriúsculas en sus caras ventrales, (7.5-)9.5-11.5(-14) × 1.4-2.9 mm, filarias internas 8, oblongas a elípticas u obovadas, 10-16.3 × (3-)5$7 \mathrm{~mm}$, color pardo en la antesis con los márgenes hialinos a amarillos, rara vez con tonos morados, obtusas en el ápice, glabras, páleas similares a las filarias internas pero más pequeñas; receptáculo plano a ligeramente convexo, 7-7.5 mm de diámetro; flores liguladas 8 , color blanquecino a rosadas o moradas, estériles aunque a veces con vestigios de gineceo, glabras, láminas elípticas, oblongas u obovadas, agudo-denticuladas, 1.8-3.6 × 1-2 cm; flores del disco 25-60, corolas tubulares, 6-9 $\times 0.7-1 \mathrm{~mm}$, color amarillo o amarillo con lóbulos color púrpura, a veces completamente púrpuras, glabras; anteras de (1.4-)3-4 mm de largo, color pardo; estilo 4.8-6.2 mm de largo, ramas del estilo con la parte proximal ensanchada, agudas en la parte distal, linear-lanceoladas, 2.5-3 $\times 1 \mathrm{~mm}$, cilioladas en el margen; cipselas lineares o linear-lanceoladas, 5.5$10 \times 1.1-2.3 \mathrm{~mm}$, surcadas longitudinalmente, color pardo oscuras a negruzcas, glabriúsculas; vilano ausente.

Distribución y ecología: especie restringida a México, se distribuye entre los $17-24^{\circ}$ de latitud norte y $96-$ $103^{\circ}$ de longitud oeste (Fig. 1B). De acuerdo con Carrasco-Ortiz (2013) y con base en nuestros resultados, Dahlia merckii habita en matorral xerófilo, bosques abiertos de Pinus, Quercus y Abies, bosque tropical caducifolio, pastizal y vegetación acuática y subacuática. Con frecuencia crece en lugares rocosos y soleados. Se le conocía de Ciudad de México, Guanajuato, Guerrero, Hidalgo, Estado de México, Michoacán, Morelos, Oaxaca, Puebla, Querétaro, San Luis Potosí, Tlaxcala y Veracruz (Carrasco-Ortiz, 2013). Además, nuestros resultados señalan su presencia en los estados de Durango y Zacatecas. De acuerdo con la regionalización de Morrone et al. (2017), Dahlia merckii crece en las provincias biogeográficas Cuenca del Balsas, Desierto Chihuahuense, Faja Volcánica Transmexicana, Sierra Madre del Sur y Sierra Madre Oriental. En Nueva Galicia está presente al sur del estado de Zacatecas en un rango de elevación de 2514-2585 m y habita en bosque de pino-encino y matorral xerófilo, de la Sierra Madre Occidental y el Desierto Chihuahuense. También 

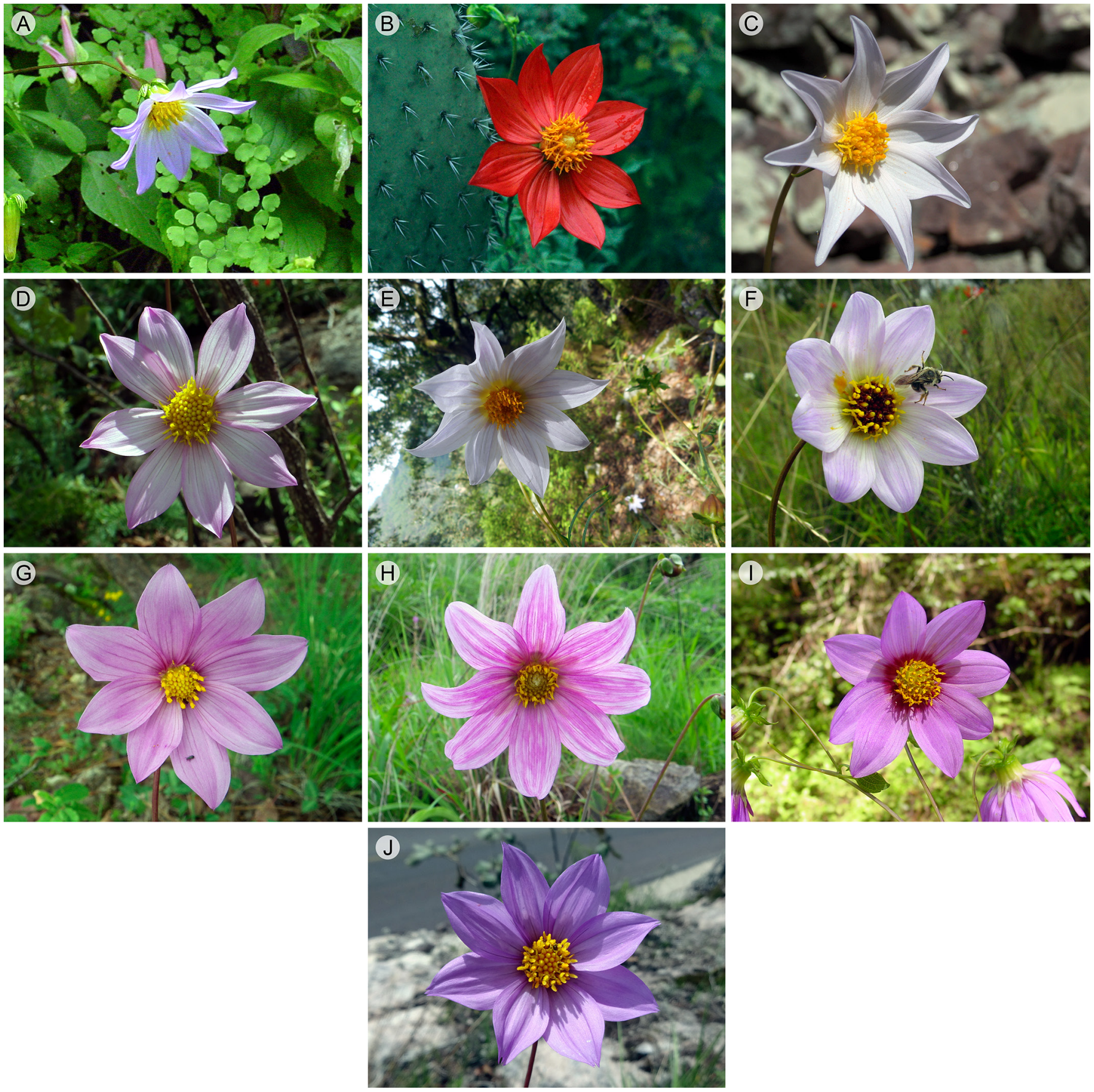

Figura 2: Riqueza del género Dahlia Cav. en la Nueva Galicia, México. A. D. barkerae Knowles \& Westc.; B. D. coccinea Cav.; C. D. merckii Lehm.; D. D. pugana Aarón Rodr. \& Art. Castro; E. D. rupicola P.D. Sørensen; F. D. scapigera (A. Dietr.) Knowles \& Westc.; G. D. sherffii P.D. Sørensen; H. D. sorensenii H.V. Hansen \& Hjert.; I. D. tenuicaulis P.D. Sørensen; J. D. wixarika Art. Castro, Carr.-Ortiz \& Aarón Rodr. Fotografías: A, F (A. Rodríguez), B, D, G, H, I (A. Castro-Castro), C (E. Rodríguez-Pérez), E, J (J. G. González-Gallegos). 
se le ha colectado al sur de Durango y norte de Zacatecas, en un rango de elevación de 2500-2600 m, en bosque de pino-encino de la Sierra Madre Occidental. Estas últimas poblaciones representan su extremo de distribución noroccidental.

Estado de conservación: Dahlia merckii presenta valores de EOO de 301,490.3 km² y de AOO de $640 \mathrm{~km}^{2}$. Con base en estos valores y de acuerdo a los criterios de UICN (2012), D. merckii puede incluirse en la categoría Preocupación Menor (LC) por su EOO y el AOO, ya que no cumple ninguno de los criterios que definen las clases En Peligro Crítico, En Peligro, Vulnerable o Casi Amenazado. En LC se incluyen taxones abundantes y de distribución geográfica amplia. Con base en nuestra experiencia en campo consideramos que no existen amenazas directas a gran escala que reduzcan las poblaciones de $D$. merckii a futuro. Más aún, hemos podido observar que con frecuencia el aclareo de bosques y la apertura de caminos favorecen el desarrollo de la especie, aunque esta reflexión debe considerarse con mesura, ya que estos fenómenos deberían de evaluarse a largo plazo.

Material examinado: MÉXICO. Ciudad de México, Ajusco, cerca de 26 millas al suroeste de la Ciudad de México a lo largo de la carretera 15 a Toluca, $2895 \mathrm{~m}$, 1.IX.1965, T. E. Melchert et al. 6238 (MEXU); Paraje de Cuauhte, 2700 m, 4.VII.1976, A. Ventura 1730 (ENCB, IBUG, MEXU); al borde de carretera, $3173 \mathrm{~m}, 29$. VI.1976, W. Wonderly 272 (MEXU, NMC). Milpa Alta, volcán Pelado, ladera este, 3334 m, 23.VIII.1987, A. Mondragón 444 (MEXU); loc. cit., 3334 m, 13.VIII.1987, A. Mondragón et al. 448 (MEXU); cerro Nepanapa a 200 $\mathrm{m}$ de la caseta pelagatos por el camino a Quinta Nepanapa, 3003 m, 15.VII.2003, J. E. Rivera et al. 3292 (MEXU); Paraje de Zompoli, 1950 m, 17.VII.1976, A. Ventura 1825 (MEXU); Mirador Santa Ana, 2700 m, 21.IX.1980, A. Ventura 3695 (CIIDIR, ENCB, IBUG, MEXU); volcán Pelado, ladera este, 3296 m, 22.VII.1986, G. Zamudio 1344 (MEXU). Tlalpan, cerca de $1 \mathrm{~km}$ al norte del límite entre el Distrito Federal y Morelos, carretera Méx. 95,

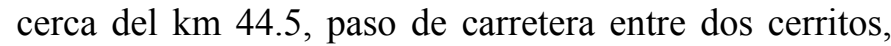
3047 m, 29.VII.1994, R. Bye et al. 19101 (MEXU); 4 km al este del Ajusco, 2750 m, 23.VI.1967, L. Gutiérrez s.n. (ENCB, IBUG, MEXU); 12 millas al norte de Tlalpan, 24 millas al norte de Cuernavaca, 3048 m, 16.VIII.1953, $W$. E. Manning 531050a (MEXU); Pedregal, cerca del Ajusco, 2554 m, 16.VII.1950, E. Matuda 19168 (MEXU); loc. cit., E. Matuda 19169 (MEXU); volcán Xitle, 2985 m, 17.IX.1950, E. Matuda 19589 (MEXU); Área Natural Protegida: Parque Ecológico de la Ciudad de México, volcán Xitle, en las faldas, al este, 2834 m, 25.VI.2002, E. Mora et al. 220 (MEXU); ejidos de Padierna, 2425 m, 16.VI.1980, M. A. Panti 106 (MEXU); parte alta del cerro de Santa Catarina, cerca de Santa Ana Astahuacán, 2604 m, 8.VII.1973, J. Rzedowski 30843 (ENCB, IBUG, MEXU); San Miguel Ajusco, 3042 m, 1.IX.1986, J. C. Soto 13044 (MEXU); ladera oriental del Xitle, $2700 \mathrm{~m}$, 17.IX.1987, J. Soto 13117 (MEXU); volcán Xitle, 3600 m, 4.VIII.1985, E. Trejo s.n. (MEXU). Durango, Durango, Predio Las Bayas de la UJED, $95 \mathrm{~km}$ al sur de Durango, 2600 m, 14.VII.1990, S. González et al. 4523 (CIIDIR). Estado de México, Amecameca de Juárez, entre el km 76 y 77 de la carretera Amecameca - Popocatépetl, 3254 m, 2.VIII.1958, J. H. Beaman 2040 (MEXU); zona de Temacoco, faldas del Parque Nacional Iztaccíhuatl Popocatépetl, 2808 m, 4.X.2012, A. Rodríguez et al. 6856 (IBUG); Barranca del Salto de Amecameca, $2475 \mathrm{~m}$, 31.VII.1881, M. Urbina s.n. (MEXU). Atlautla de Victoria, entre el km 8 y 9 de la carretera que va de Amecameca hacia Paso de Cortés en las faldas del volcán Popocatépetl, 2850 m, 5.X.2012, M. A. Carrasco et al. 86 (IBUG); San Pedro Nexapa, sobre la entrada hacia el arroyo Las Palomas entre el km 10 y 11 de la carretera que va de Amecameca hacia Paso de Cortés, en las faldas del Popocatépetl, 3004 m, 5.X.2012, M. A. Carrasco et al. 93 (IBUG). Chalco, cerro de Jocotitlán, 2354 m, 11.VII.1954, E. Matuda 31003 (MEXU); cerca de la Colonia Agrícola Manuel Ávila Camacho, 2750 m, 11.IX.1966, A. M. Pascoe 239 (ENCB, IBUG); ladera este del cerro La Tijera, $10 \mathrm{~km}$ al sureste de Santa Ana, $2800 \mathrm{~m}, 10$.VIII.1969, $A$. Pineda 831 (CIIDIR, ENCB). Coatepec Harinas, Llano 
Grande, 2700 m, 15.VI.1951, E. Matuda 21453 (MEXU); cerca de Río Frío, 2986 m, 28.VII.1944, A. J. Sharp 44100 (MEXU). Ecatepec de Morelos, a lo largo de la carretera de peaje Cuernavaca - Ciudad de México, cerca del km 20 al norte de Cuernavaca, 2403 m, 23.X.1970, A. J. Cronquist et al. 10830 (MEXU). Huehuetoca, cerros al norte de Huehuetoca, límite con el estado de Hidalgo, 2444 m, 12.VIII.1951, D. B. Gold 21770 (MEXU); Huehuetoca, 2262 m, 6.IX.1953, E. Matuda 29070 (MEXU); cerro Ahumada, cerca del Rancho Nuevo, $4 \mathrm{~km}$ al norte de Huehuetoca, 2342 m, 11.VIII.1971, J. Rzedowski 28388 (MEXU). Ixtapaluca, entre el km 40 y 41 de la carretera libre 150 México - Puebla en los bordes del parque nacional Zoquiapan, 3119 m, 7.X.2012, M. A. Carrasco et al. 118 (IBUG); Estación Experimental de Investigación y Enseñanza de Zoquiapan, $8 \mathrm{~km}$ al sur de Río Frío, cerro del Espinazo cerca de medio $\mathrm{km}$ al oeste de Llano Coleto, 3594 m, 18.VII.1975, S. D. Koch et al. 75342 (MEXU); Estación Experimental de Investigación y Enseñanza de Zoquiapan, $8 \mathrm{~km}$ al sur de Río Frío, camino 5, entre $\mathrm{km} 0$ y 1, adelante del entronque con camino 7, $3285 \mathrm{~m}, 1$. IX.1978, R. Vega 474 (MEXU). Ixtapan de la Sal, carretera México - Puebla $8 \mathrm{~km}$ al sureste de Río Frío, orilla de arroyo, 3055 m, 8.VII.1978, J. García s.n. (MEXU); cercanía de Ixtapan de la Sal, 1800 m, 16.VIII.1953, E. Matuda et al. 28784 (MEXU). Juchitepec, cerro Dos Cerros, $10 \mathrm{~km}$ al sureste de Milpa Alta, $2850 \mathrm{~m}, 22$.VII.1982, F. G. Lorea 2162 (MEXU). La Paz, cerro de La Caldera, 2400 m, 15.VII.1945, D. B. Gold 763 (MEXU). Ocoyoacac, vía férrea al lado norte del pueblo, sobre cerro, cerca de la carretera Panorámica, en escarpamiento a un lado de la vía férrea, $2640 \mathrm{~m}, 7$. VIII.2001, H. Vibrans 7524 (MEXU). Ocuilan, alrededores del poblado de Lagunillas, $5 \mathrm{~km}$ al norte de Santa Martha, carretera Tenango Lagunas de Zempoala, 2799 m, 27.IX.2009, A. Rodríguez et al. 6015 (IBUG); Lagunas de Zempoala, por la carretera México 4, entre Santa Martha y Tres Marías, 2902 m, 27.IX.2009, A. Rodríguez 6017 (IBUG). Texcalyacac, 4 $\mathrm{km}$ al nor-noroeste de Texcalyacac, $2653 \mathrm{~m}, 5 . \mathrm{VII} .1989$, Y. López 23 (MEXU). Texcoco, carretera Texcoco - Apizaco, $17 \mathrm{~km}$ al este de Texcoco, $2760 \mathrm{~m}, 1$.VIII.1978, C.
Perino et al. 3410 (CHAPA, IBUG). Tlalmanalco, $3 \mathrm{~km}$ al este de San Rafael, 2800 m, 10.VIII.1969, N. Aragón 68 (ENCB, IBUG); Llano Atlihuiyán, $10 \mathrm{~km}$ al este de San Rafael, 3204 m, 18.VIII.1982, J. Rzedowski 37870 (MEXU). Tultitlán, Sierra de Guadalupe, al norte de Cuautepec, 2494 m, 30.VII.1950, D. B. Gold s.n. (MEXU). Guanajuato, Guanajuato, alrededores de la mina San Gregorio, cerca de El Chorro, 2100 m, 29.X.1987, J. Rzedowski 45529 (IBUG, IEB, MEXU). San José Iturbide, $500 \mathrm{~m}$ al norte de La Mesa a $3 \mathrm{~km}$ de San Diego, cerca de las minas, 2403 m, 6.VIII.2002, J. Gutiérrez et al. 367 (MEXU). San Miguel de Allende, cerro Alcocer, cerca de El Palo Huérfano, 2183 m, A. Mora 798 (IEB). Tierra Blanca, pico rocoso situado al norte de las instalaciones de Televisa, 3329 m, L. S. Calderón et al. 118 (IEB), 126 (IEB), 127 (IEB); ladera este del cerro Zamorano, 2817 $\mathrm{m}$, M. Gómez et al. 646 (IEB). Victoria, aproximadamente $5.5 \mathrm{~km}$ al norte de Joya Fría, 2379 m, E. Pérez et al. 3394 (IEB); cerro El Rosario, por Joya Fría, 2454 m, E. Ventura et al. 9696 (IEB). Xichú, cerro El Escobillal, Sierra de Xichú, 1900 m, E. Ventura et al. 9579 (IEB). Hidalgo, Ajacuba, Rincón del Tecojote, al sur del poblado de Ajacuba, vertiente norte de la Sierra del Monte Alto de Temoaya, ejido Ajacuba, 2426 m, 22.VIII.1993, I. Díaz et al. 1318 (MEXU). Epazoyucan, Cerro Grande, $3 \mathrm{~km}$ al sur de Epazoyucan, 2700 m, 1.VIII.1971, J. Rzedowski 28329 (CIIDIR, ENCB). Mineral del Chico, Peña del Cuervo, extremo oeste de la Sierra de Pachuca, $2781 \mathrm{~m}$, 3.X.2005, E. R. Chaires 2 (MEXU); Rincón de Las Brujas, 2400 m, 21.IX.1988, L. Zamora et al. 467 (CIIDIR, ENCB). Mineral del Monte, carretera Real del Monte Mineral del Chico, 2824 m, 16.IX.2011, A. Rodríguez et al. 6457 (IBUG). Omitlán de Juárez, km 17 carretera Méx. 105 entre Pachuca y Huejutla, 2548 m, 16.IX.2011, A. Rodríguez et al. 6453 (IBUG). Pachuca de Soto, 1.5 $\mathrm{km}$ al noreste de Pachuca, 2750 m, 15.VI.1975, M. Medina 418 (ENCB, IBUG); entre el km 8 y 9 de la carretera México 105 entre Pachuca y Huejutla, antigua carretera a El Chico, 2279 m, 12.IX.2007, A. Rodríguez et al. 5260 (IBUG); ejido El Cerezo (El Llano), entre el km 7 y 8 de la carretera a El Chico a partir de la carretera México 105, 
2930 m, 12.IX.2007, A. Rodríguez et al. 5273 (IBUG). Singuilucan, Cuyamaloya, $3 \mathrm{~km}$ al nor-noroeste de Matías Rodríguez, 2559 m, 21.VII.1988, M. Medina 3647 (MEXU). Zimapán, km 4 de la brecha Trancas - Nicolás Flores, 2430 m, 13.IX.2011, A. Rodríguez et al. 6394 (IBUG); entre el km 145 y 146 de la carretera Méx. 85 entre Pachuca y Tamazunchale, 2264 m, 14.IX.2011, A. Rodríguez et al. 6410 (IBUG). Michoacán de Ocampo, Maravatío, cerro El Calvario, San Miguel, $2960 \mathrm{~m}, H$. Díaz et al. 6827 (IEB). Tlalpujahua, cerro Peña Blanca, ejido San Francisco de los Reyes, 2738 m, S. Zamudio et al. 3967 (IEB). Morelos, Cuernavaca, autopista Cuernavaca - México, El Mirador, 2261 m, 25.VIII.1968, L. M. González 1804 (IBUG); cerca de Cuernavaca, $1803 \mathrm{~m}$, 26.VII.1951, E. Matuda 21476 (MEXU); campos de lava al norte de Cuernavaca, 2403 m, 5.VI.1904, C. G. Pringle 13039 (MEXU); carretera México - Cuernavaca, 3004 m, 10.VII.1971, L. Wolfgang 1839 (MEXU). Huitzilac, volcán Las Palomas, norponiente del estado de Morelos, corredor biológico Ajusco - Chichinautzin, 3193 m, 22. XI.2007, T. Amezcua 6 (MEXU), loc. cit., 2753 m, $T$. Amezcua 7 (MEXU); sobre la carretera a Lagunas de Zempoala, 2819 m, 25.VII.1975, anónimo 147 (MEXU); carretera Zempoala - Huitzilac entre el km 4 y 11, 2825 m, 28.VII.1987, J. Castañeda 227 (MEXU); autopista México - Cuernavaca km 53, 2510 m, 25.X.1959, J. Espinosa 217 (MEXU); entre el km 2 y 3 al norte de Tepoztlán, sobre la cañada de la zona arqueológica del Tepozteco, 1825 m, 5.X.1986, G. Flores et al. 153 (MEXU); Parque Nacional Lagunas de Zempoala, 3004 m, 13. VIII.1985, D. López 674 (MEXU); loc. cit., 2820 m, 29. VII.1949, J. D. Mitchell 33 (MEXU); autopista Cuernavaca - México $\mathrm{km} 1$ al noroeste de La Pera, $2331 \mathrm{~m}$, 6.VIII.1984, M. A. Morales 420 (MEXU); Parque Nacional Lagunas de Zempoala, al noreste de Huitzilac, 2835 m, 31.VII.1988, R. Ramírez et al. 907 (IBUG); alrededores del poblado de Cuajomulco, 2576 m, 28.IX.2009, $A$. Rodríguez et al. 6022 (IBUG); a $5 \mathrm{~km}$ al oeste de Huitzilac, rumbo a las Lagunas de Zempoala, $2843 \mathrm{~m}, 18$. XI.1981, J. L. Villaseñor 149 (MEXU); a 5.5 millas al oeste, camino al Parque Nacional, por la carretera 95,
2844 m, 22.X.1981, M. J. Warnock 2573 (MEXU). Tepoztlán, km 9.25 al oeste de Tres Cumbres (Méx. 95), autopista Cuernavaca - México, El Mirador, 2200 m, 25. VIII.1968, L. M. Villarreal 1804 (IBUG). Oaxaca, Tepelmeme, Distrito de Coixtlahuaca, 2843 m, 18.VII.1985, $P$. Tenorio 9323 (MEXU). Puebla, Atlixco, campo experimental de San Juan Tetla, Barranca de Cotzala, 3104 m, 26.VIII.1965, A. May 922 (MEXU). Cuyoaco, Zona Arqueológica de Cantona, $10 \mathrm{~km}$ al noroeste de Tepeyahualco, a $3 \mathrm{~km}$ de Tezontepec, camino a Texcal, $2507 \mathrm{~m}$, 2.VIII.2002, A. García et al. 7403 (MEXU). Chalchicomula de Sesma, Ciudad Serdán, 2577 m, 15.VIII.2009, F. Ventura 2119 (MEXU). Guadalupe Victoria, Sierra de Mextaloyan, km 20 de la carretera Perote - Los Humeros, 2800 m, 26.VII.1986, J. A. Pérez et al. 1172 (IBUG). Puebla, Parque Estatal General Lázaro Cárdenas, 2328 m, 18.VII.1995, M. Martínez 710 (MEXU). San Salvador el Seco, Rincón Quelite, entre Salvador el Seco y Alchichica, 2393 m, 28.VII.1967, R. M. Rosas 476 (MEXU). Tehuacán, $15.7 \mathrm{~km}$ al suroeste de San Bartolo Teontepec, 2102 m, 7.IX.2005, O. Téllez et al. 19267 (MEXU). Tlahuapan, Puente del Emperador, La Venta, $2551 \mathrm{~m}$, 25.VIII.1944, A. J. Sharp 44542 (MEXU). Zacapoaxtla, 5 km al sur de San José del Progreso, 2424 m, 4.VIII.1998, J. L. Contreras 5558 (HUAP, MEXU). Querétaro, Amealco de Bonfil, cerca de San Bartolo, 8 km al este de Amealco, sobre la carretera a Acapulco, 2497 m, 15.VIII.1989, J. Rzedowski et al. 48650 (IBUG, IEB, MEXU, QMEX). Colón, cerro Zamorano, en la parte alta del macizo montañoso a $500 \mathrm{~m}$ del puerto del Carmen, en el pico ubicado al suroeste de las instalaciones de Televisa, $3293 \mathrm{~m}, L$. S. Calderón 64 (IEB); pico rocoso situado al este de las instalaciones de Televisa, 3293 m, L. S. Calderón et al. 162 (IEB); cerro Zamorano, camino de Los Trigos a las antenas de Televisa, parte baja, $2677 \mathrm{~m}, L$. S. Calderón et al. 290 (IEB); loc. cit., parte media del cerro, $2904 \mathrm{~m}$, 19.X.1998, L. S. Calderón et al. 315 (IBUG, IEB, QMEX); Puerto del Carmen, 3293 m, E. Pérez et al. 4180 (IEB); parte más alta del cerro Zamorano, $3270 \mathrm{~m}$, 13.VI.1971, J. Rzedowski et al. 426 (ENCB, MEXU); parte alta del cerro Zamorano, $3211 \mathrm{~m}, 1 . I X .1987$, J. Rze- 
dowski 44413 (MEXU, QMEX, UAT); loc. cit., 3250 m, J. Rzedowski 47888 (IEB). Landa de Matamoros, cerro Piedra de la Cruz, noreste de Pinalito, 2478 m, 7.IX.1990, E. Carranza 2728 (IBUG, IEB, QMEX). Pinal de Amoles, $2 \mathrm{~km}$ al norte de El Madroño, cerro La Calentura, 2864 m, 13.IX.1989, E. Carranza 2084 (CIIDIR, IEB, MEXU, QMEX); Puerto Agua Blanca, aproximadamente $15 \mathrm{~km}$ al sur de Pinal de Amoles, $2451 \mathrm{~m}$, 5.IX.1985, $R$. Fernández 3078 (ENCB, MEXU); cerro Boludo, al norte de Puerto de Los Velázquez, por la brecha a San Gaspar, 2858 m, E. Pérez 468 (IEB); loc. cit., 2904 m, 11.VII.2006, E. Pérez 4687 (IEB, QMEX). Tolimán, alrededores de El Derramadero, 2550 m, 9.IX.1990, J. Rzedowski 50069 (IBUG, IEB, QMEX). San Luis Potosí, San Luis Potosí, Álvarez, 2221 m, 28.IX.1902, E. Palmer 211 (MEXU). San Nicolás Tolentino, carretera 70 al este de San Luis Potosí, oeste de Santa Catarina, 2700 m, 15.VIII.1991, J. A. Soule et al. 2612 (CIIDIR, MEXU). Zaragoza, $21 \mathrm{mi}-$ llas al este de la carretera 57 hacia Río Verde, 2353, 10. IX.1967, T. E. Melchert et al. 67-13 (MEXU); Villa de Zaragoza, poblado de San Francisco de Álvarez, 2278 m, 12.IX.2011, A. Rodríguez et al. 6375 (IBUG). Tlaxcala, Sanctórum de Lázaro Cárdenas, carretera Calpulalpan Apizaco entre el km 76 y 77 cerca de la desviación Españita y San Miguel Tepalca, 2621 m, 3.X.2012, M. A. Carrasco et al. 80 (IBUG). Veracruz, Acultzingo, Cumbres de Acultzingo, 2176 m, 18.IX.1962, R. Hernández 4 (MEXU); Cumbres de Acultzingo, camino a Puente Colorado, límites de Veracruz y Puebla, 2303, 15.IX.1987, J. L. Martínez et al. 956 (IBUG, MEXU, XAL); brecha Boca del Monte - El Sumidero, la brecha inicia en el km 27.5 de la carretera Tehuacán - Orizaba, 2415 m, 16. IX.2006, A. Rodríguez et al. 5983 (IBUG); Puerto del Aire km 35 carretera Orizaba - Tehuacán, 2750 m, 22. XI.1967, M. Rosas 795 (MEXU). Maltrata, Maltrata, 1730 m, 12.V.1937, E. Matuda 1363 (MEXU, XAL). Perote, al sureste de la Hacienda de San Agustín, 2545 m, 4.XI.1998, G. Castillo 18684 (MEXU); al norte de La Gloria, 2686 m, 18.XI.1998, G. Castillo 18945 (MEXU). Soledad Atzompa, Col. Rancho Nuevo, congregación Atzompan, 2162 m, 8.XI.1985, J. L. Martínez et al. 1069
(MEXU, XAL). Zacatecas, Guadalupe, cerro La Virgen (cerro de las Antenas) al sur de la ciudad, $2585 \mathrm{~m}, 5$. VIII.2015, E. J. Rodríguez 1466 (CIIDIR). Saín Alto, Sierra de Chapultepec a $2.5 \mathrm{~km}$ al suroeste de la carretera Fresnillo - Saín Alto, 2557 m, 7.X.2017, E. J. Rodríguez 7098 (CIIDIR).

Dahlia rupicola P.D. Sørensen, Rhodora 71: 346. 1969. TIPO: MÉXICO. Durango, about $11 \mathrm{mi} \mathrm{W}$ of El Soldado along route 40, $1828 \mathrm{~m}$, T. E. Melchert et al. 6306 (holotipo: IA!, isotipos: DEK!, TEX!, WIS!). Figs. 1B, 2E.

Subarbusto, 0.6-1.5 m de alto; sin raíces fasciculado-tuberosas; tallos leñosos y con frecuencia ensanchados en sus bases, 1.5-2(-10) $\mathrm{cm}$ de diámetro, glabros y pruinosos; internudos 4-16 cm de largo; pecíolo sólido, $0.6-8.3 \times 0.08-0.5 \mathrm{~cm}$; hojas ovadas en contorno general, (9.6-)20-35 × 4.2-24.6 cm, bipinnatisectas; estipelas presentes en la base del primer y segundo par de folíolos, 3-12 × 2-4.8 cm; folíolos 5-11, 4.6-18 × 2.7-7 cm, los tres pares inferiores presentan peciólulos, $0.6-3 \mathrm{~cm}$ de largo, linear-atenuados, uninervados, ápice acuminado, obtuso a redondeados en la base, herbáceos, concoloros, color verde pálido, glabriúsculos, margen entero; lóbulos de los folíolos alternos, 1.6-8 × 0.8-3.8 cm, linear-atenuados; cabezuelas 2-5 por planta, 7-9.5 cm de diámetro; pedúnculos 6-11(-22) cm de largo, glabros; involucro crateriforme, filarias externas 5-8, erectas o ascendentes en la antesis, color verde, hialinas, ovado-lanceoladas, glabriúsculas en sus caras ventrales, 9-14 × 4-8.5 mm, filarias internas 8 , ovado-lanceoladas, $16-22 \times 6-8.5 \mathrm{~mm}$, color amarillo-pardas y con numerosas nervaduras pardorojizas, acuminadas en el ápice, glabras, páleas similares a las filarias internas pero más pequeñas; receptáculo plano o convexo, 8.7-10.5 mm de diámetro; flores liguladas 8 , color lila y amarillo-maculadas en sus bases, estériles pero con vestigios de gineceo, glabriúsculas, láminas ovadas, agudas-denticuladas, $2.2-4 \times 0.7-2 \mathrm{~cm}$; flores del disco 85-109, corolas tubulares, 6.8-12.4 × 1-2 mm, color amarillo, glabras; anteras 1.9-3.8 mm de largo, color 
amarillo-anaranjadas; estilo 7.2-9.4 $\mathrm{mm}$ de largo, ramas del estilo con la parte proximal ensanchada, agudas en su parte distal, linear-lanceoladas, 3.6-4.6 × 0.8-1.1 mm, cilioladas en el margen; cipselas oblongo-rectangulares, (8.8-)10-12 × 1.5-2 mm, surcadas longitudinalmente, color pardo oscuras a negruzcas, pubescentes, tuberculadas; vilano ausente.

Distribución y ecología: Dahlia rupicola se conocía solo de su localidad tipo y sus alrededores, en los declives hacia el Pacífico de la porción central de la Sierra Madre Occidental (SMO) en Durango (Sørensen, 1969; Carrasco-Ortiz, 2013). Aquí damos a conocer una población disyunta encontrada a $360 \mathrm{~km}$ en línea recta de la localidad tipo en el extremo sur de la SMO en Zacatecas (Fig. 1B). Los registros amplían el rango de distribución de la especie entre $\operatorname{los} 21-23^{\circ}$ de latitud y $103-105^{\circ}$ de longitud. Dahlia rupicola se desarrolla en un rango de elevación de 1828-2682 m. Llama la atención la plasticidad de sus requerimientos ambientales, puesto que se conocía que habitaba en bosque de pino-encino, pero la población encontrada en Zacatecas se desarrolla en bosque tropical caducifolio. En ambas localidades, las plantas crecen en grandes acantilados sobre suelos rocosos.

Estado de conservación: Dahlia rupicola presenta valores de EOO de $2153.794 \mathrm{~km}^{2}$ y de AOO de $24 \mathrm{~km}^{2}$. Con base en estos valores y siguiendo los criterios de UICN (2012), D. rupicola puede considerarse como una especie En Peligro (EN, criterios B1a, B2a y B2c(ii)).

Material examinado: MÉXICO. Durango, Espinazo del Diablo, km 165 de la carretera Méx. 40 Durango a Mazatlán, 2470 m, 24.X.2017, A. Castro et al. 4366 (CIIDIR, IBUG, MEXU); El Salto, cerca de 15 millas al este de El Palmito, 16 millas al oeste de la ciudad sobre la carretera 40, 1829 m, 5.IX.1966, T. E. Melchert et al. 6312 (DEK, F); Espinazo del Diablo, km 164 de la carretera Méx. 40 entre Durango y Mazatlán, 18 km al Oeste de La Ciudad, $1 \mathrm{~km}$ al oeste de Los Bancos, 2682 m, 6.IX.2010, A. Rodríguez et al. 6133 (IBUG). San Dimas, La Fraguita,
$1 \mathrm{~km}$ al oeste de la población y $1 \mathrm{~km}$ antes de La Mesa Verde, 2328 m, 15.X.2016, J. G. González et al. 2191 (CHAP, CIIDIR, IBUG). Zacatecas, García de la Cadena, El Abra, camino de descenso al rancho El Zapote desde el pueblo de García de la Cadena, 1855 m, 25.VIII.2014, A. Castro et al. 3838 (CIIDIR, IBUG, MEXU); loc. cit., 14.VIII.2017, A. Castro et al. 4294 (CIIDIR).

\section{DIsCUSIÓN}

Villaseñor (2016) reportó a Dahlia merckii para Ciudad de México, Guanajuato, Guerrero, Hidalgo, Jalisco, Estado de México, Michoacán, Morelos, Nuevo León, Oaxaca, Puebla, Querétaro, San Luis Potosí, Tamaulipas, Tlaxcala, Veracruz y Zacatecas. Sin embargo, consideramos que algunos de estos reportes se deben a información desactualizada. Por ejemplo, las poblaciones registradas para la porción norte de la Sierra Madre Oriental en los estados de Nuevo León y Tamaulipas fueron reconocidas como una especie distinta, D. tubulata P.D. Sørensen (Sørensen, 1980). En otro caso, un trabajo actualizado sobre las asteráceas en el estado de Jalisco no reporta a $D$. merckii (Harker, 2017). El trabajo de Villaseñor (2016) es una contribución invaluable para el conocimiento de la fitodiversidad y punto de partida para nuevos enfoques en la investigación botánica mexicana. Sin embargo, la ausencia de una lista de ejemplares analizados o la imposibilidad de acceso libre a los datos de respaldo no nos ha permitido comprobar sus resultados. De este modo, consideramos que las novedades aquí mostradas tienen un buen sustento como primer reporte de la especie para la región de la Nueva Galicia.

Ninguna especie de Dahlia está registrada en la Lista Roja de la UICN (2017). Sin embargo, en la Norma Oficial Mexicana (NOM-059-SEMARNAT-2010) se encuentran D. scapigera y D. tenuicaulis, ambas bajo la categoría Protección Especial (Pr) (SEMARNAT, 2010). Las especies registradas en este informe, D. merckii y D. rupicola, podrían ser incluidas en la Norma Oficial Mexicana. Por otro lado, y considerando los resultados del análisis biogeográfico de Carrasco-Ortiz (2013), recomendamos que 14 especies de Dahlia que tienen una dis- 
tribución geográfica limitada y no se encuentran en áreas naturales protegidas, sean incluidas en las normas mexicanas. Dahlia es un grupo casi exclusivo de México y un recurso importante en la horticultura ornamental (INEGI, 1998), por estas razones recomendamos la realización de un estudio para evaluar su estado de conservación y la propuesta de categorías de protección a nivel nacional e internacional. Por último, la ampliación de las distribuciones de $D$. merckii y $D$. rupicola también extienden la amplitud adaptativa de ambas a condiciones ecológicas diferentes a las conocidas, y señala la necesidad de continuar e impulsar el trabajo de exploración botánica en México.

\section{CONTRIBUCIÓN DE AUTORES}

ACC, PCR y RGMF diseñaron la investigación. ACC, CJRD, JGGG y PCR llevaron a cabo el trabajo de campo y las recolectas. ACC identificó el material. ACC y RGMF escribieron el texto. Todos los autores corroboraron las identificaciones, contribuyeron a la discusión, revisión y aprobación del manuscrito final.

\section{FINANCIAMIENTO}

Estudio apoyado por el Consejo Nacional de Ciencia y Tecnología a través del Programa Cátedras CONACyT.

\section{AgradeCIMIENTOS}

Agradecemos al personal asociado de las colecciones botánicas consultadas, a dos revisores anónimos y a los miembros del comité editorial por los valiosos comentarios a las versiones iniciales del manuscrito. Gracias a Aarón Rodríguez Contreras y Emmetth Rodríguez Pérez por facilitarnos el uso de sus fotografías e información de sus recolectas botánicas.

\section{LITERATURA CITADA}

Bachman, S., J. Moat, A. W. Hill, J. de la Torre y B. Scott. 2011. Supporting Red List threat assessments with GeoCAT: geospatial conservation assessments tool. ZooKeys 150: 117-126. DOI: https://doi.org/10.3897/ zookeys.150.2109
Carrasco-Ortiz, M. A. 2013. Distribución geográfica y riqueza del género Dahlia Cav. (Asteraceae) en México. Tesis de licenciatura. Centro Universitario de Ciencias Biológicas y Agropecuarias, Universidad de Guadalajara. Guadalajara, Jalisco, México. 76 pp.

Castro-Castro A., A. Rodríguez, G. Vargas-Amado y M. Harker. 2012. Diversidad del género Dahlia (Asteraceae: Coreopsideae) en Jalisco, México y descripción de una especie nueva. Revista Mexicana de Biodiversidad 83: 347-358. DOI: https://dx.doi.org/10.22201/ ib.20078706e.2012.2.941

Castro-Castro A., O. Zuno-Delgadillo, M. A. Carrasco-Ortiz, M. Harker y A. Rodríguez. 2015. Novedades en el género Dahlia (Asteraceae: Coreopsideae) en Nueva Galicia, México. Botanical Sciences 93(1): 41-51. DOI: https:// doi.org/10.17129/botsci.239

ESRI. 2002. ArcView 3.3. Environmental Systems Research Institute, Inc. Redlans, USA.

Harker, M. 2017. Las compuestas (familia Asteraceae o Compositae). In: Cruz, A. A., A. Ordorica, J. Valero y E. D. Melgarejo (coords.). La biodiversidad en Jalisco. Estudio de Estado. Volumen II. Comisión Nacional para el Conocimiento y Uso de la Biodiversidad (CONABIO) y Secretaría de Medio Ambiente y Desarrollo Territorial (SEMADET). Cd. Mx., México. Pp. 143-149.

INEGI. 1998. La horticultura ornamental en México. Instituto Nacional de Estadística Geografía e Informática. Aguascalientes, México. $81 \mathrm{pp}$.

JSTOR. 2017. JSTOR Global Plants. http://plants.jstor.org/ (consultado noviembre de 2017).

McVaugh, R. 1984. Compositae. In: Anderson, W. (ed.). Flora Novo-Galiciana. A Descriptive Account of the Vascular Plants of Western Mexico. Vol. 12. The University of Michigan Press. Ann Arbor, USA. 1157 pp.

Moreno, N. P. 1984. Glosario botánico ilustrado. Compañía Editorial Continental S.A. de C.V. Cd. Mx., México. 300 pp.

Morrone, J. J., T. Escalante y G. Rodríguez-Tapia. 2017. Mexican biogeographic provinces: Map and shapefiles. Zootaxa 4277(2): 277-279. DOI: https://doi.org/10.11646/ zootaxa.4277.2.8 
Rzedowski, J. y R. McVaugh. 1966. La Vegetación de la Nueva Galicia. Contributions from the University of Michigan Herbarium 9: 1-123.

Rzedowski J. y G. Calderón de Rzedowski. 2008. Compositae Tribu Heliantheae I (géneros Acmella - Jefea). Flora del Bajío y de regiones adyacentes 157: 1-344.

SEMARNAT. 2010. Norma Oficial Mexicana NOM-059SEMARNAT-2010, Protección ambiental-Especies nativas de México de flora y fauna silvestres-Categorías de riesgo y especificaciones para su inclusión, exclusión o cambio-Lista de especies en riesgo. Secretaría del Medio Ambiente y Recursos Naturales. Diario Oficial de la Federación. Cd. Mx., México. http://dof.gob.mx/nota detalle.php? codigo $=5173091 \&$ fecha $=30 / 12 / 2010$

Sørensen, P. D. 1969. Revision of the genus Dahlia (Coreopsideae, Heliantheae-Coreopsidinae). Rhodora 71: 309-365, 367-416.

Sørensen, P. D. 1980. New taxa in the genus Dahlia (Asteraceae, Heliantheae-Coreopsidinae). Rhodora 82: 353-360.
Thiers, B. 2017. Index Herbariorum: a global directory of public herbaria and associated staff. New York Botanical Garden's, Virtual Herbarium. http://sweetgum.nybg.org/ ih/ (consultado noviembre de 2017).

UICN. 2012. Categorías y criterios de la Lista Roja de la Unión Internacional para la Conservación de la Naturaleza Versión 3.1. 2a. ed. Gland, Suiza y Cambridge, Reino Unido. vi +34 pp.

UICN. 2017. The IUCN Red List of Threatened Species, versión 2017-2. http://www.iucnredlist.org (consultado noviembre de 2017).

Villaseñor, J. L. 1991. Las Heliantheae endémicas a México: una guía hacia la conservación. Acta Botanica Mexicana 15: 29-46. DOI: https://doi.org/10.21829/abm15.1991.619

Villaseñor, J. L. 1993. La familia Asteraceae en México. Revista de la Sociedad Mexicana de Historia Natural 44: 117-124.

Villaseñor, J. L. 2016. Checklist of the native vascular plants of Mexico. Revista Mexicana de Biodiversidad 87: 559-902. DOI: https://dx.doi.org/10.1016/j.rmb.2016.06.017 